\title{
The Calculation Approach for the Setting of Precautionary Areas of Offshore Platforms Based on Turning Ability of Vessels
}

\author{
Tian Bo \\ Haiyun College, SHAN DONG Jiao Tong University \\ Weihai, P. R. China \\ tianbowh@163.com
}

\section{Lv Mingshan}

School of Navigation, Wuhan University of Technology

Wuhan, P. R. China

lumingshan11@163.com

\author{
Deng Lin \\ School of Navigation, Wuhan University of Technology \\ Wuhan, P. R. China \\ whutdlm@163.com
}

\begin{abstract}
As the development of maritime resources with growing enthusiasm, quantity of offshore platforms are put into use. However, the installation of offshore platforms tends to impede navigation and fishing activities. To ensure the safety of platforms and nearby vessels, it is necessary to set the precautionary areas which have a broad range for offshore platforms, we propose one approach named "Tactical Diameter Method" to calculate the range for the precautionary areas based on the turning ability of the vessel. We calculate the distance between the initial position of one vessel and safety zones through tactical diameter, after adding the influence of wind, current and the distance for buffer, we finally get the range of precautionary areas. By using our approach, we can set the precautionary areas for the offshore platform effectively.
\end{abstract}

Keywords: offshore platform, range, the precautionary areas, turning ability, tactical diameter,

\section{INTRODUCTION}

Along with the onshore resources are gradually drying up, the development of offshore resources has been attached more and more importance, more and more offshore platforms play the vital role in the resource exploitation. And nearby waters of offshore platforms often has routes used or catchments areas, for the protection of the marine environment, ensure the safety of platforms and nearby vessels, it is needed to set safety zones and precautionary areas which have a broad range, the shape of the safety zones is circle, the shape of the precautionary areas is ring, safety zones and precautionary areas are in the center of offshore platform. Generally

\author{
Wang Yusheng \\ School of Navigation, Wuhan University of Technology \\ Wuhan, P. R. China \\ wangyusheng1990@126.com \\ Ma Yong* \\ School of Navigation, Wuhan University of Technology \\ Hubei Key Laboratory of Inland Shipping Technology \\ Wuhan, P. R. China \\ *Corresponding Author
}

speaking, the vessel cannot enter safety zones absolutely ; In contrast, precautionary areas relaxed a lot adjacent to safety zones, vessels can sail in precautionary areas, but the officers on duty should pay much attention to their navigation, strength the lookout, ensure that the vessel can away from waters of offshore platforms rapidly, in order to prevent vessels endanger the safety of offshore platforms, which dues to operation difficulties caused by bad weather, mechanical failure and other factors, ensure the safety of vessels when there have accidents in platforms, so precautionary areas can be understand as buffer zones for offshore platforms and safety zones, when vessels enter the precautionary areas a warn can be issued to vessels from platforms which remind vessels' drivers of strengthening lookout, driving carefully, handling vessels to keep a safe distance from platforms to prevent vessels entering the safety zones blindly, or ensure that vessels can take necessary measures to protect themselves and platforms.

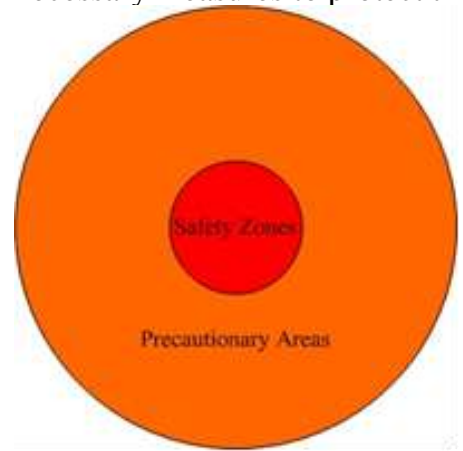

Figure 1.Safety zones and precautionary areas 
According to the provision of the item 5 in article 14 of the United Nations Convention on the law of the sea and requirements of British health and Safety Executive, the radius of the safety zones should not be more than 500 meters; however the design of the precautionary areas' range lacks of relevant standards domestic, articles can be referenced are less and less. For example, "Offshore Oil Safety Management Rules" promulgated by the State Bureau of Safe Production Supervision and Administration in 2009 doesn't mention safety zones and precautionary areas, rules only regular that stand-by vessels must protect platforms in platforms nearby waters' the range of which is $5 \mathrm{n}$ miles. When relevant organizations conduct navigation safety assessments, they often refer to the way of Elgin wellhead platform \{March 2007, Elgin wellhead platform away from Aberdeen about 150 miles occurred an accident of gas leaking, gas emitted at a speed about $2 \mathrm{~kg} / \mathrm{s}$, the platforms are exposed to the flammable, toxic hydrocarbons, the accident resulted in total 238 peoples in Elgin wellhead platform and its nearby Rowan Viking drilling rig evacuated, and around two platforms is delineated danger zones by 2 miles $\}$, while at the angle of preventing collision, solved this problem by introducing the concept of DCPA. In narrow waters, DCPA is 0.5-2 n miles generally, while in open waters, so domestic organizations usually design precautionary areas as a ring with the radius of $2 \mathrm{n}$ miles. The rationality and effectiveness of this approach are ready to be tested, so it is of great importance to research on the setting of precautionary areas of offshore platforms. Therefore, we propose one approach named "Tactical Diameter Method" for the setting of precautionary areas of offshore platforms based on turning ability of vessels.

\section{METHOD INTRODUCTION}

\section{A. Calculation of the minimum range of precautionary areas}

Our approach assumes that if a vessel is navigating at a constant speed in front of a platform, the vessel conducts emergency steering to avoid entering the safety zones, the vessel cycles with hard, when the movement of the vessel tangent to the edge of safety zones, distance between initial position and the offshore platform but subtract the radius of safety zones is the minimum radius of precautionary areas, the precautionary areas is in the shape of ring. Part of the movement of the vessel tangent to the edge of safety zones is an arc whose diameter is the tactical diameter of vessel. It is assumed that the vessel should be the standard forms or the biggest vessels of waters near the platform.

The calculation method is shown in Figure 2.

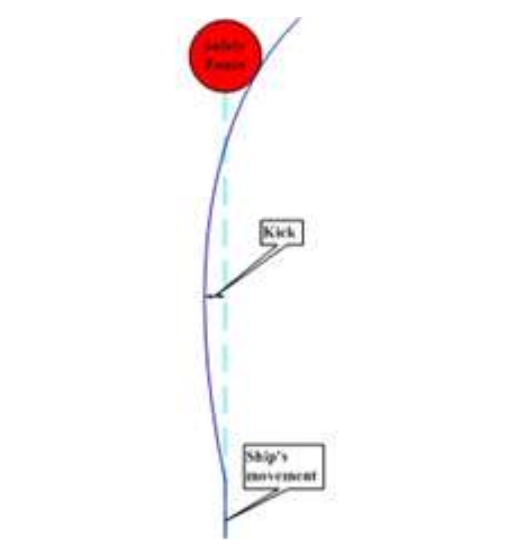

Figure 2.The principle of formula

Calculation formula:

$$
S=0.7 * D_{T}+\sqrt{\left(\frac{D_{T}}{2}+r\right)^{2}-\left(\frac{D_{T}}{2}-0.01 L\right)^{2}}-r
$$

(1)

$K \_$Coefficient, 0.041;

$S$ _ The range of precautionary areas;

$D_{T}$

$D_{T} \_$Tactical diameter of vessel;

$r$ _ The radius of safety zones;

$L$ _Length;

The formula divides precautionary areas into two parts; one part is the longitudinal distance from the initial position to the center of vessel's movement, the other part is the longitudinal distance from the center of vessel's movement to platform. The difference between advance and radius of movement of vessel is the longitudinal distance from initial position to the center of vessel's movement (about 1/2 times tactical diameter), advance of vessels about 0.6 1.2 times more than tactical diameter of vessels, here is 1.2 times $D_{T}$, so the longitudinal distance from initial position to the center of movement of vessel is 0.7 times $D_{T}$; After vessel hard to port or hard to starboard there will has kick which is about 0.01times as long as length of the , the lateral distance from the center of vessel's movement to platform is the difference between 0.5 times tactical diameter and the kick, so the longitudinal distance from the center of movement to platform is

$$
\sqrt{\left(\frac{D_{T}}{2}+r\right)^{2}-\left(\frac{D_{T}}{2}-0.01 L\right)^{2}} \text {. }
$$

\section{B. After joining the effects of wind and current of special precautionary areas range correction}

Formula 1 is the preliminary formula for calculating the range of precautionary areas. It is the premise of the formula to calculate the influence of the platform areas where there is no the effects of wind and current, but it is impossible that there is no wind and current in the actual situation, so we need to modify the formula 1 . Specifically the longitudinal distance will decrease when vessel under the condition of against the wind and current, and is advantage for vessel to turn 180 degrees; However the 
longitudinal distance will be increased when vessel under the condition of downwind downstream. Therefore, the appropriate calculation should be carried out under the condition of downwind and downstream to ensure the reliability of precautionary areas' range. After considering the wind and current effect, the formula is:

$$
S^{\prime}=S+\Delta S_{a}+\Delta S_{w}
$$

Among them $\Delta S_{a}$ is the additional moving distance under the effects of wind, $\Delta S_{w}$ is the additional moving distance under the effects of current. The calculation formula of both can respectively refer to the formulas of wind-induced drift as well as the flow-induced to drift, the specific formula is:

$$
\Delta S_{a}=K\left(\frac{B_{a}}{B_{w}}\right)^{1 / 2} \cdot e^{-0.14 V_{S}} \cdot V_{a} \cdot T
$$

In the formula:

$K$-coefficient 0.041 ;

$B_{a}$ - The windward area of upper hull waterline $\left(\mathrm{m}^{2}\right)$;

$B_{w}$ - The side area of under hull waterline $\left(\mathrm{m}^{2}\right)$;

$V_{s}$ - Speed (kn);

$V_{a}$ - The relative wind speed $(\mathrm{m} / \mathrm{s})$;

$T$ - Drift time (s)

$$
\Delta S_{w}=V_{C} * T * 0.8
$$

In the formula:

$$
V_{c} \text { - Velocity of flow }(\mathrm{m} / \mathrm{s}) \text {, according to }
$$
the data displayed on chart;

$\mathrm{T}$ - Drift time (s).

The drift time of vessel is steering from the beginning to the tangent between the edge of vessel motion trajectory and the safety, but it is difficult to obtain much more accurate drift time. So considering the simplicity of calculation, $T$ can take $1 / 2$ of turning time, turning time is the amount of time the vessel turning 360 degrees. During the turning the vessel will continue to slow down, until the turning circle is steady, the drop of the speed of vessel will be $40 \% \sim 50 \%$, the very large oil tanker even reached $65 \%$. Therefore $\mathrm{T}$ take $1 / 2$ of the actual turning time will be large than vessel's drift time. It improves the security of precautionary areas. In general, the turning time of the 10000 -ton class vessel is 6 minutes; the very large vessel's turning time is greater than 12 minutes.

\section{THE APPLICATION OF COMPUTING}

Take platforms in the East China Sea coast for example, the area locates between China Eastern road and foreign sea road, generally the water depth is more than 60 meters, and very large vessels can past. The maximum summer wind speed is $30.39 \mathrm{~m} / \mathrm{s}$, the direction of the wind is SSW, tide stream is $1 \mathrm{knot}$, the velocity is $2 \mathrm{~m} / \mathrm{s}$ if there is the kurtosis. To "Maersk. Mike - Kinney. Muller" container vessel for calculating vessel type, the total length of 397.7 meters, 58.5 meters wide, the maximum draft is 16.02 meters, take constant speed at sea 21 knots as speed, The tactical diameter take 6.5 times total length of the vessel, drift time take 6 minutes. Thanks to more vessels in the vicinity of the sea area, the radius of the offshore platform safety should take a maximum of 500 meters.

So the calculation process of the minimum offshore platform precautionary areas range is:

$$
\begin{aligned}
& S=0.7 * 6.5 * 400+\sqrt{(6.5 * 400 \div 2+500)^{2}+(6.5 * 400 \div 2-0.01 * 400)^{2}} \\
& -500=2569.2 m \\
& \Delta S_{a}=0.041 * \sqrt{4} * e^{-0.14 * 0.515 * 21 *(30.39-0.515 * 21) * 6} \\
& * 60=127 m \\
& \Delta S_{w}=(0.515+2) * 6 * 60 * 0.8=724.3 m
\end{aligned}
$$

The minimum offshore platform precautionary areas range is:

$$
S^{\prime}=S+\Delta S_{a}+\Delta S_{w}=3420.5 m=1.847 \text { nmile }
$$

\section{SAFETY RECOMMENDATIONS}

Above calculation result under the assumption that the vessel suddenly found the offshore platform exists in front and hard a port (starboard) emergency measures must be taken to avoid running into the safety zones, It is the minimum value of the precautionary areas of theory, considering it takes time to take corresponding measures when the vessel received a warning or found platforms ahead. Therefore, it is recommended that precautionary areas offshore platform in the set on the basis of the theoretical calculated value $0.5 \mathrm{n}$ mile expansion, namely the final value of precautionary areas' range should be:

$$
S^{\prime \prime}=S^{\prime}+0.5 \text { nmile }=2.347 \mathrm{nmile}
$$

Sailing with caution when the vessel sailing into the precautionary areas. And special measures must take to ensure the safety of the platform and vessel when the vessel is about to enter the precautionary areas.

\section{CONCLUSIONS}

We propose a new method to calculate the range of the precautionary areas based on the turning ability of the vessel, we assume that movement of the vessel tangent to the edge of safety zones after the vessel hard to port or hard to starboard. Then we calculate the minimum range of precautionary areas through tactical diameter of vessel, after adding the influence of wind, current and the distance for buffer, we finally get the range of precautionary areas. Effectiveness of our approach is verified by the numerical examples.

\section{ACKNOWLEDGMENT}

This research was financially supported by the National Natural Science Foundation of China (Grant NO.51309186) and the Fundamental Research Funds for Central University (Grant NO.WUT:2013-IV-015).

\section{REFERENCES}

[1] China MSA .The Demonstration for the Effect of Safety of Navigation and the Measures for the Evaluation-Administration of 
Water and Underwater Activities of the People's Republic of China [Z]. 2011-07-01;

[2] Ministry of Transport of the People's Republic of China. Regulations on Navigation Safety Management of Water and Underwater Activities of the People's Republic of China [Z]. 201103-01;

[3] Standing Committee of the National People's Congress of the People's Republic of China. Maritime Traffic Safety Law of the People's Republic of China [Z]. 1984-01-01;

[4] The International Maritime Organization. International Regulations for Preventing Collisions at Sea, 2007 edition [Z]. 2010;

[5] State Administration of Work Safety of the People's Republic of China. Rules of Safety Management of Offshore Oil[Z]. 2011;
[6] IALA Recommendation O-139 on the Marking of Man-Made Offshore Structures, 2008 edition [Z]. 2008-11-01;

[7] Health and Safety Executive .Safety Zones around Oil and Gas Installations in Waters around the UK [Z]. 2007;

[8] Standing Committee of the National People's Congress of the People's Republic of China. Marine Environment Protection Law of the People's Republic of China [Z]. 2000-04-01;

[9] Li Song, Zhou Jiang -hua, Xu Ting-liu, Bao Xiong-guan. Optimal Design of precautionary area Routing Based on Navigational Traffic Conflict Technique [J]. Navigation of China, 2013, 36(1):95-100 ;

[10] WU Hui-guo, LI Song, XIAO Ying-jie. Safety Assessment of Ship's Routing Precautionary Area Based on VTCT [J].Port\&Waterway Engineering, 2012, (6):58-68 